Editorial

Pensar en Movimiento:

Revista de Ciencias del Ejercicio y la Salud ISSN 1659-4436

Vol. 10, No.1, pp. 1- 5

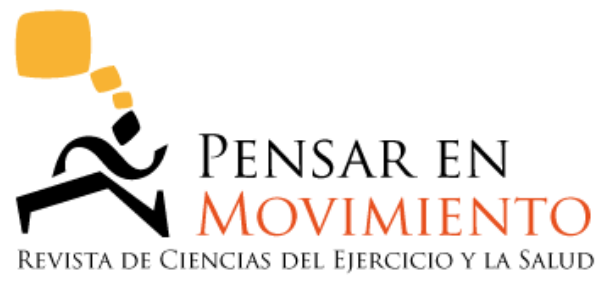

\title{
EL INGREDIENTE EXPERIENCIA EN EL APRENDIZAJE UNIVERSITARIO (PERSPECTIVA DE UN EDUCADOR FÍSICO)
}

"No aprendemos oyendo, aprendemos haciendo"

Dr. Eric Mazur

Conferencia en la Universidad de Costa Rica

Agosto del 2010.

\section{RESUMEN}

Aragón-Vargas, L.F. (2012). El ingrediente experiencia en el aprendizaje universitario (perspectiva de un educador físico. Pensar en Movimiento: Revista de Ciencias del Ejercicio y la Salud, 10 (1), 15. Se presenta en este editorial un ensayo de reflexión sobre el papel que juegan las experiencias en el proceso de aprendizaje de los estudiantes universitarios. Se aprovecha una pequeña colección de anécdotas para ilustrar algunos conceptos básicos del aprendizaje por experiencias: el peligro de los aprendizajes incompletos que dan una falsa sensación de seguridad; la importancia de la pregunta fascinante y cómo se traduce esta en la enseñanza de destrezas en educación física y deportes; el poder de las emociones en el proceso de aprendizaje; la extensión de las experiencias más allá de las destrezas involucradas y la necesidad de complementar las experiencias con la reflexión, asimilación y desarrollo de modelos, donde la persona que tiene el papel docente debe ser siempre intencional, orientada a los logros.

Palabras claves: Aprender haciendo, educación universitaria, estilos de aprendizaje

\section{ABSTRACT}

Aragón-Vargas, L.F. (2012). The Experiential Ingredient in College Learning (A Physical Educator's Perspective). Pensar en Movimiento: Revista de Ciencias del Ejercicio y la Salud, 10 (1), 1-5. This editorial is an essay, reflecting on the role that experiences play in the learning process of college students. A small collection of anecdotes is used to illustrate a few basic concepts of experiential learning: the danger of incomplete learning resulting in a false sense of security; the importance of the intriguing question and how that intriguing question translates into the teaching of sports and physical education skills; the power of emotions in the learning process; the extension of experiences beyond the particular skills involved; and the need to supplement experiences with reflection, assimilation and model development, where the person with a teaching role needs to be always intentional or goaloriented.

Key Words: Experiential learning, college education, learning styles 
Alejandro Nerdrick, del periódico costarricense La Teja, captó magistralmente el momento en su titular de la página 6 del día 9 de junio de 2011: "La gran lección de su vida: Estudiantes de rescate acuático reciben clase de verdad". Aún trastornado por los acontecimientos del día anterior y agotado después de dormir muy poco, yo me preguntaba: "¿cómo nos metimos en esto? ¿Es realmente necesario complicarse tanto la vida con las clases?"

En realidad, el periodista no estaba exagerando, pues con un grupo de 22 estudiantes de la Universidad de Costa Rica, un colega y yo habíamos programado una práctica de rescate en ríos para el curso de Salvamento Acuático. Hacia el final de la práctica ocurrió un accidente, en el cual estuvimos a punto de perder a una alumna. La experiencia quedó profundamente grabada en todos los involucrados: la desaparición de M.I. entre las fuertes corrientes color café con leche del río Reventazón, la búsqueda desesperada, el rescate del cuerpo sin vida, la ventilación boca a boca, la impresionante respuesta -digna de un libro de texto- de todos los estudiantes, la llamada al 9-1-1, el arribo de los oficiales de policía que venían "a levantar el cuerpo", las preguntas de los periodistas, la entrega de M.I. sana y salva a su papá... Al repasar dos días después todo lo acontecido, en nuestra cómoda y segura aula de clase, verificamos que todos íbamos a necesitar bastante tiempo para procesar los acontecimientos. La experiencia había sido tan fuerte y tan completa que hasta me sentí tentado a eximir del examen final a todo el grupo.

La reflexión sobre esta y algunas otras experiencias importantes de mi vida como profesor, poco después de cumplir 30 años de iniciar mi labor de docencia universitaria, me permitió identificar la existencia de un patrón bastante claro en mi estrategia docente, el cual pareciera dar buenos resultados: se trata de incorporar elementos clave del aprendizaje por experiencia de David A. Kolb (1981), a quien no había leído sino hasta hace poco. Por eso decidí aceptar la invitación de la Vicerrectoría de Docencia de la universidad a escribir el presente ensayo, aunque me costó hacer las paces con lo paradójico de intentar comunicar el poder de la experiencia por un medio escrito.

Curiosamente, mi incursión en estos esfuerzos por ofrecer experiencias más palpables a mis estudiantes también se originó en un contexto acuático: durante un paseo en Palo Seco (Pacífico Central de Costa Rica), un amigo y yo fuimos arrastrados por la corriente en el mar. Yo no me asusté, pues tenía entrenamiento como guardavidas y estaba en buena condición física, pero rescatar a mi amigo me resultó considerablemente más difícil de lo esperado. Claro, todas mis clases de salvamento acuático se habían desarrollado en una piscina bajo techo, en un ambiente totalmente controlado; por lo tanto, yo había aprendido destrezas sumamente útiles, pero a la vez adquirí una falsa sensación de confianza, como puede ocurrir fácilmente en los profesionales graduados en otras disciplinas de nuestras universidades. En el caso particular de mi formación como guardavidas faltó el enfrentamiento a nuevas situaciones en otros contextos.

Ante esto, decidí exponer a mis estudiantes a situaciones más realistas. Nuestra primera gira (1987) fue de participación voluntaria, en un día feriado, viajamos en el microbús Volkswagen de mis papás. Fuimos a playas de Doña Ana, coordinamos con un guardavidas local, y la experiencia fue muy exitosa. Tanto que a partir de esa fecha se incluyó en el cronograma del curso la práctica de rescate en el mar, aunque hemos optado por hacerla en un ambiente un poco más retante y a la vez 
con mayor apoyo, en playas de Jacó. Recientemente se incluyó también la práctica de rescate en ríos, en vista del alto porcentaje de ahogamientos que ocurren en Costa Rica en ese contexto.

La pregunta fascinante. Es inspirador observar a un grupo de futuros misioneros, convencidos de su vocación, en el proceso de estudiar un idioma extranjero: el potencial para el aprendizaje es ideal debido a su actitud de total disposición, pues saben que están adquiriendo herramientas para solucionar problemas concretos y relevantes. Para ellos, el reto de comunicarse de manera efectiva con las personas a quienes van a servir los involucra emocionalmente en el aprendizaje; se sabe que este es más profundo cuando incluye las emociones (Medina, 2009). El reconocido maestro Dr. Kenneth Bain le llama a esto, a un nivel muy específico, the intriguing question, la pregunta o el problema fascinante (Bain, 2004). En sus investigaciones con grandes maestros, Bain concluye que "Los seres humanos tienen la mayor probabilidad de aprender a profundidad cuando están intentando resolver problemas o contestar preguntas que han llegado a considerar como importantes, fascinantes, o hermosos" (2009, p.11). Más aún, según el autor, es más probable que los estudiantes lleguen a cuestionar y modificar sus paradigmas cuando estos producen explicaciones incorrectas 0 no satisfactorias a sus intentos por resolver esas preguntas o problemas.

En la Educación Física y la Recreación se utiliza a menudo una versión muy práctica de la pregunta fascinante: se trata de los retos o "acertijos" prácticos, tales como escalar una pared o cruzar un puente de cuerdas bajo condiciones que tendrán consecuencias relevantes para los participantes, pero también se trata de los desafíos específicos de una enorme variedad de destrezas motrices como lanzar, atrapar, manipular, derribar, desplazarse, entre otras. Estas pruebas tienen un elemento de experiencia mucho más concreto y que fácilmente las hace más relevantes a los estudiantes, quienes están en mejor posición de modificar sus paradigmas cuando estos producen resultados no satisfactorios. Las preguntas acertadas en Química o Filosofía son los problemas o retos en Educación Física.

Así, la naturaleza de la enseñanza de destrezas en la Educación Física calza con el precepto de aprender haciendo. Pero no se debe caer en la trampa de pensar que solamente en ese ámbito vale la pena este tipo de aprendizaje, pues las experiencias refuerzan algunos conceptos de un modo que la mejor explicación teórica quizás no lograría. Los profesionales de la recreación, representados con excelencia por la Asociación Cristiana de Jóvenes (conocida como YMCA, por sus siglas en inglés) y los Scouts, han utilizado las experiencias del juego y el deporte desde finales del siglo XIX para enseñar no solamente las destrezas propiamente dichas, sino los principios morales, cívicos y religiosos; lo cual se puede experimentar a plenitud en los campamentos. En su artículo de 1984, El campamento como recurso educativo, Aragón cita a Robert S. Sabean al decir que "el campamento es un laboratorio de aprendizaje extraordinario, porque reúne lo práctico-teórico como pocas otras instituciones educativas" ( p. 128).

En nuestro caso, por lo menos, desde 1987 hemos organizado prácticas de laboratorio de fisiología del ejercicio en el Volcán Irazú (a 3432 m.s.n.m.) para ilustrar los efectos agudos de la altitud sobre el rendimiento físico; esta experiencia fue publicada por Aragón Vargas (1989), y su efectividad se puede ilustrar con la reacción de un estudiante quien, con una sensación de asfixia causada por la presencia de gases de azufre en el aire, no podía creer cuánto lo afectaba la altitud. A pesar de explicarle que sus síntomas no obedecían a la altura, posiblemente la experiencia haya 
quedado registrada en su mente como "efectos de la altura sobre el rendimiento físico". Este tipo de experiencias es importante en un campo de estudio en el cual, en las palabras de Aragón y Fernández (1995) aún:

(. . .) está sembrado de 'autoridades' que emiten sus argumentos demagógicos sin fundamento científico, de mitos y sofismas que son invocados con frecuencia a conveniencia del sujeto, de figuras que alcanzan el éxito gracias al azar y, a veces, aún a pesar de los métodos de entrenamiento utilizados. (p. 14).

Para inclinar la balanza a favor del trabajo científico y sistemático es necesario aportar argumentos consistentes, enseñar teorías sólidas y brindar oportunidades para que los estudiantes tengan experiencias que se manejen con el mismo rigor utilizado en la investigación científica.

Los cursos de capacitación que impartí durante varios años a nutricionistas del Gatorade Sports Science Institute ${ }^{\circledR}$, a partir de 1998, incluyeron distintas versiones de este elemento. Estas nutricionistas se encargaban de enseñar sobre la importancia de la hidratación durante la actividad física en escuelas, colegios y equipos deportivos en Centroamérica, Colombia y México, en el contexto de un esfuerzo conocido como Mercadeo Profesional (algo similar a los programas de educación de higiene buco-dental de la Colgate ${ }^{\circledR}$ en Costa Rica). Sin embargo, su formación generalmente era débil en nutrición deportiva; más aún, algunas de ellas probablemente no practicaban deporte ni sabían lo que era deshidratarse por sudoración. Por ello, incorporé los talleres de sudoración a las sesiones de capacitación; en la versión más sencilla, todas participaban en una clase de ejercicio de una hora en un ambiente caliente y húmedo, la cual culminaba con la oportunidad de tomarse una bebida deportiva fría. La versión más sofisticada consistía en retarlas a beber suficiente líquido durante la sesión de ejercicio en el calor para compensar las pérdidas por sudoración, ni más ni menos; los resultados se verificaban cuidadosamente mediante la medición del peso corporal antes y después de la actividad física, de la ingesta de líquido y de la eliminación de orina. Siempre hubo varias participantes sorprendidas por los resultados, pero algunas veces el asombrado fui yo. Todas esas circunstancias constituyeron oportunidades para cuestionar o modificar paradigmas.

El denominador común en estas historias es la importancia de aprender haciendo, de incluir experiencias concretas y retantes, tanto en los cursos prácticos como en los teóricos, para fomentar la profundidad y la amplitud del aprendizaje. Pero, ¿es suficiente para los estudiantes el "pasar por la experiencia"? Aparentemente no. En el curso La Universidad del Siglo XXI, de la Enseñanza al Aprendizaje, organizado por primera vez por la Universidad de Costa Rica y LASPAU/Harvard, en el año 2010, mi principal aprendizaje fue la importancia de ser intencional. Un buen profesor debe ser intencional, es decir, lo que hace debe tener un propósito concreto que ayuda al aprendizaje. Para ello, debe tener muy claros los objetivos de cada experiencia. Además, las actividades especiales deben involucrar activamente a todos los estudiantes, evitando convertirlos en espectadores pasivos de un gran espectáculo. El profesor debe esforzarse por hacer el proceso completo, en otras palabras, no solamente facilitar las experiencias y el aprendizaje activo, sino también la reflexión, la formación de conceptos abstractos y la puesta a prueba de las implicaciones (Haynes, 2007). De esta manera facilitará la creación de modelos en sus estudiantes, a la vez que les ayudará a enamorarse, esto es, a involucrarse emocionalmente con sus objetos de aprendizaje (Papert, 1980). 
Regresando a la pregunta fascinante que me hacía yo después de nuestra experiencia de rescate acuático en el río Reventazón, me atrevo a responder que sí: si queremos fomentar el aprendizaje amplio y profundo en nuestros estudiantes es necesario complicarnos la vida con las clases -sin dejar de tomar todas las medidas del caso para que el aprendizaje sea seguroreconociendo que el proceso implica renunciar al control absoluto que normalmente preferimos mantener en el aula.

Luis Fernando Aragón Vargas, Ph.D., FACSM

Editor en Jefe

\section{Referencias}

Aragón Vargas, L. F. y Fernández Ramírez, A. (1995). Fisiología del Ejercicio: respuestas, entrenamiento y medición. San José, Costa Rica: Editorial de la Universidad de Costa Rica,

Aragón Vargas, L. F. (1989). Enseñanza-aprendizaje en fisiología del ejercicio. Revista Educación, 13(1-2), 131-139.

Aragón Vargas, L. F. (1984). El campamento como recurso educativo. Revista Educación, 8(1-2), 127-130.

Bain, K. (2004). What Makes Great Teachers Great? The Chronicle of Higher Education The Chronicle Review, 50(31), B7.

Bain, K. (Spring, 2009). Understanding Great Teaching. Association of American Colleges \& Universities Peer Review, 9-12.

Haynes, C. (2007). Experiential Learning - Learning by Doing. (Revisado por Taylor, A. en 2008). Recuperado en

http://adulteducation.wikibook.us/index.php?title=Experiential Learning - Learning by Doing

Kolb, D. A. (1981). Learning styles and disciplinary differences. En A. Chickering (Ed.), The Modern American College. San Francisco, California: Jossey-Bass Inc., Publishers. pp. 232-255.

Medina, J. (2009). Brain Rules. Seattle, Washington: Pear Press.

Papert, S. (1980). Mindstorms: Children, Computers, and Powerful Ideas. New York: Basic Books.

\section{(c) (3) $\Theta$}

Esta obra está bajo una

licencia Creative Commons Atribución-NoComercial-SinDerivadas 3.0 Costa Rica 